\title{
Influenza and Other Respiratory Viruses Involved in Severe Acute Respiratory Disease in Northern Italy during the Pandemic and Postpandemic Period (2009-2011)
}

\author{
Elena Pariani, ${ }^{1,2}$ Marianna Martinelli, ${ }^{1}$ Marta Canuti, ${ }^{3}$ \\ Seyed Mohammad Jazaeri Farsani, ${ }^{3,4}$ Bas B. Oude Munnink, ${ }^{3}$ \\ Martin Deijs, ${ }^{3}$ Elisabetta Tanzi, ${ }^{1,2}$ Alessandro Zanetti, ${ }^{1,2}$ \\ Lia van der Hoek, ${ }^{3}$ and Antonella Amendola ${ }^{1,2}$ \\ ${ }^{1}$ Department of Biomedical Sciences for Health, University of Milan, Via C. Pascal 36, 20133 Milan, Italy \\ ${ }^{2}$ CIRI-IT, Department of Health Sciences, University of Genoa, Via A. Pastore 1, 16132 Genoa, Italy \\ ${ }^{3}$ Laboratory of Experimental Virology, Department of Medical Microbiology, Center for Infection and Immunity Amsterdam \\ (CINIMA), Academic Medical Center of the University of Amsterdam, Meibergdreef 15, 1105 AZ Amsterdam, The Netherlands \\ ${ }^{4}$ Tehran University of Medical Sciences, 16 Azar Avenue, Enghelab Square, Tehran 1417614411, Iran \\ Correspondence should be addressed to Marianna Martinelli; marianna.martinelli@unimi.it
}

Received 11 February 2014; Revised 26 May 2014; Accepted 27 May 2014; Published 12 June 2014

Academic Editor: Rudolf K. Braun

Copyright (C) 2014 Elena Pariani et al. This is an open access article distributed under the Creative Commons Attribution License, which permits unrestricted use, distribution, and reproduction in any medium, provided the original work is properly cited.

\begin{abstract}
Since 2009 pandemic, international health authorities recommended monitoring severe and complicated cases of respiratory disease, that is, severe acute respiratory infection (SARI) and acute respiratory distress syndrome (ARDS). We evaluated the proportion of SARI/ARDS cases and deaths due to influenza A(H1N1)pdm09 infection and the impact of other respiratory viruses during pandemic and postpandemic period (2009-2011) in northern Italy; additionally we searched for unknown viruses in those cases for which diagnosis remained negative. 206 respiratory samples were collected from SARI/ARDS cases and analyzed by realtime RT-PCR/PCR to investigate influenza viruses and other common respiratory pathogens; also, a virus discovery technique (VIDISCA-454) was applied on those samples tested negative to all pathogens. Influenza A(H1N1)pdm09 virus was detected in $58.3 \%$ of specimens, with a case fatality rate of $11.3 \%$. The impact of other respiratory viruses was $19.4 \%$, and the most commonly detected viruses were human rhinovirus/enterovirus and influenza A(H3N2). VIDISCA-454 enabled the identification of one previously undiagnosed measles infection. Nearly $22 \%$ of SARI/ARDS cases did not obtain a definite diagnosis. In clinical practice, great efforts should be dedicated to improving the diagnosis of severe respiratory disease; the introduction of innovative molecular technologies, as VIDISCA-454, will certainly help in reducing such "diagnostic gap."
\end{abstract}

\section{Introduction}

Most cases of influenza A(H1N1)pdm09 infection have a mild outcome; however some present as severe acute respiratory infection (SARI) and require admission to intensive care unit (ICU) $[1,2]$. The main reason for admission to ICU is a pulmonary inflammatory syndrome characterized by diffuse alveolar damage (acute respiratory distress syndrome: ARDS), which can be fatal. Since the beginning of 2009 pandemic, international health authorities recommended monitoring severe and complicated cases of influenza infection $[3,4]$. Considering the serious outcome of these respiratory diseases, the contribution of other respiratory pathogens besides $\mathrm{A}(\mathrm{H} 1 \mathrm{~N} 1)$ pdm09 should be envisaged [5]. Additionally, in clinical practice, a specific causative agent which explains the respiratory symptoms is often unidentified, owing to the lack of sensitive tests or the presence of an asyet unknown pathogen. The recently developed VIDISCA454 (VIrus DIScovery using CDNA Amplified fragment-length polymorphism combined with Roche-454 high-throughput sequencing) is a sensitive sequence-independent virus discovery technique which can be used to reveal as-yet unknown viruses $[5,6]$. 
This study aimed at evaluating the proportion of SARI/ARDS cases and deaths due to A(H1N1)pdm09 infection and assessing the impact of other respiratory pathogens during pandemic and postpandemic period (2009-2011) in northern Italy as well as searching for unknown viruses in those cases for which diagnosis remained negative. To this end, common respiratory pathogens were investigated and VIDISCA-454 methodology was applied on samples which remained negative for all tested pathogens.

\section{Materials and Methods}

In the capacity of reference laboratory operating within InfluNet network [7], our laboratory is in charge of carrying out the virological surveillance of severe forms of influenza infection in Lombardy (nearly 10 million inhabitants). From October 1, 2009, to April 30, 2011, 206 respiratory samples were collected from patients hospitalized due to severe respiratory illness. Of these, $61.2 \%$ were males with a median age of 44.3 years (IQR: 49.7 years; range: 1 month-89 years); $17.5 \%$ were children $\leq 5$ years and $23.3 \%$ were $\geq 65$ years. Data on comorbidities presence were available for nearly $70 \%$ of study patients: $64.3 \%$ reported medical conditions $[3,4]$; in detail, $25.6 \%$ had weakened immune system (due to cancer, HIV/AIDS, or long-term steroid treatment), $19.7 \%$ heart disease, $11.6 \%$ asthma/chronic lung disease, and $10.4 \%$ neurological/neurodevelopmental conditions. Out of 206 patients, 91 (59.3\% males; $18.7 \%$ aged $\leq 5$ years, $58.2 \%$ aged 6-64 years) were SARI cases who required admission to ICU and extracorporeal membrane oxygenation (ECMO) therapy, and 115 (62.6\% males; $16.5 \%$ aged $\leq 5$ years, $60 \%$ aged $6-$ 64 years) were ARDS cases, as defined by the European Consensus Conference [8]. Nine ARDS patients (median age: 35.6 years, IQR: 21.4 years) died during hospitalization: case fatality rate (CFR) in our ARDS series was 7.8\% (9/115). No SARI case was fatal.

Respiratory specimens (paired nasal/oral swab and bronchoalveolar lavage) were collected from each SARI/ARDS case. Nucleic acids were purified by NucliSENS easyMAG (bioMérieux, France) and analyzed by real-time RT-PCR assay to identify influenza virus. In detail, a one-step realtime RT-PCR assay was performed to simultaneously detect influenza viruses type A and B [9]. The subtyping of influenza A positive samples was carried out by a one-step realtime RT-PCR assay using specific primer/probe sets for the hemagglutinin gene [10].

The clinical specimens that resulted negative to influenza virus detection were then screened by real-time RTPCR/PCR for a panel of respiratory pathogens (Respiratory MWS r-gene Real-time PCR, bioMérieux, France) to detect respiratory syncytial virus (RSV) A and B; human metapneumovirus (hMPV) A and B; human rhinovirus (hRV) and enterovirus (hEV); adenovirus (AdV); human bocavirus (hBoV) 1-4; human coronavirus (hCoV) 229E, NL63, OC43, HKU1; human parainfluenza virus (hPIV) 1-4; Chlamydophila pneumoniae; Mycoplasma pneumoniae.

Cases which resulted negative to all diagnostic assays were further investigated by VIDISCA-454 technique. This
TABLE 1: Impact of respiratory pathogens on the patients with SARI/ARDS $(N=206)$.

\begin{tabular}{lccc}
\hline & \multicolumn{3}{c}{ Number of positive samples } \\
Pathogen & SARI & ARDS & SARI/ARDS \\
& $(N=91)$ & $(N=115)$ & $(N=206)$ \\
\hline Influenza & 58 & 62 & 120 \\
A(H1N1)pdm09 virus & 7 & 4 & 11 \\
hRV/hEV & 7 & 1 & 8 \\
Influenza A(H3N2) virus & 3 & 1 & 4 \\
RSV & 2 & 2 & 4 \\
hCoV & 2 & 2 & 4 \\
hPIV & 2 & 0 & 2 \\
AdV & 1 & 0 & 1 \\
Influenza B virus & 1 & 0 & 1 \\
hBoV & 0 & 0 & 0 \\
hMPV & 0 & 0 & 0 \\
Chlamydophila & 0 & 0 & 0 \\
pneumoniae & 2 & 3 & 5 \\
Mycoplasma pneumoniae & 0 & & \\
\hline Coinfections & & &
\end{tabular}

is a virus discovery method based on recognition of restriction enzyme cleavage sites, ligation of adaptors, and subsequent amplification by PCR combined with high-throughput sequencing 454 FLX/Titanium system (Roche, USA) [5].

\section{Results}

Influenza $\mathrm{A}(\mathrm{H} 1 \mathrm{~N} 1) \mathrm{pdm} 09$ virus was detected in $58.3 \%$ $(120 / 206)$ of SARI/ARDS cases $(61.7 \%$ males; $13.3 \%$ aged $\leq 5$ years, $67.5 \%$ aged 6-64 years). Moreover, the presence of another condition possibly increasing the risk for developing influenza-related complications [3, 4] was acknowledged for nearly half of $\mathrm{A}(\mathrm{H} 1 \mathrm{N1})$ pdm09-positive SARI/ARDS cases: $25.4 \%$ had weakened immune system, $15.2 \%$ had heart disease, $11.9 \%$ were morbidly obese people (body mass index $\geq 40$ ), $10.2 \%$ had asthma/chronic lung disease or neurological/neurodevelopmental conditions, and 4.2\% were pregnant women. Approximately half (62/120: $51.7 \%)$ of $\mathrm{A}(\mathrm{H} 1 \mathrm{~N} 1)$ pdm09-positive patients had ARDS. It is worth mentioning that $\mathrm{A}(\mathrm{H} 1 \mathrm{~N} 1) \mathrm{pdm} 09$ was identified in $77.8 \%(7 / 9)$ of fatal ARDS cases $(42.9 \%$ males, median age: 30.4 years, IQR: 15.4 years). Four (4/7: 57.1\%) of these individuals belonged to risk categories (i.e., two were cancer patients, one was morbidly obese, and one had underlying neurodevelopmental conditions). Thus, the A(H1N1)pdm09 CFR was $11.3 \%$ (7/62).

The impact of respiratory pathogens other than A(H1N1)pdm09 was $19.4 \%$ (40/206) (65\% males; $30 \%$ aged $\leq 5$ years, $47.5 \%$ aged $6-64$ years). $\mathrm{HRV} / \mathrm{hEV}$ were the most frequently identified viruses followed by influenza $\mathrm{A}(\mathrm{H} 3 \mathrm{~N} 2)$ virus, accounting for $27.5 \%(11 / 40)$ and $20 \%(8 / 40)$ of infections, respectively (Table 1$)$. No fatal cases were ascribable to pathogens other than $\mathrm{A}(\mathrm{H} 1 \mathrm{~N} 1) \mathrm{pdm} 09$. 


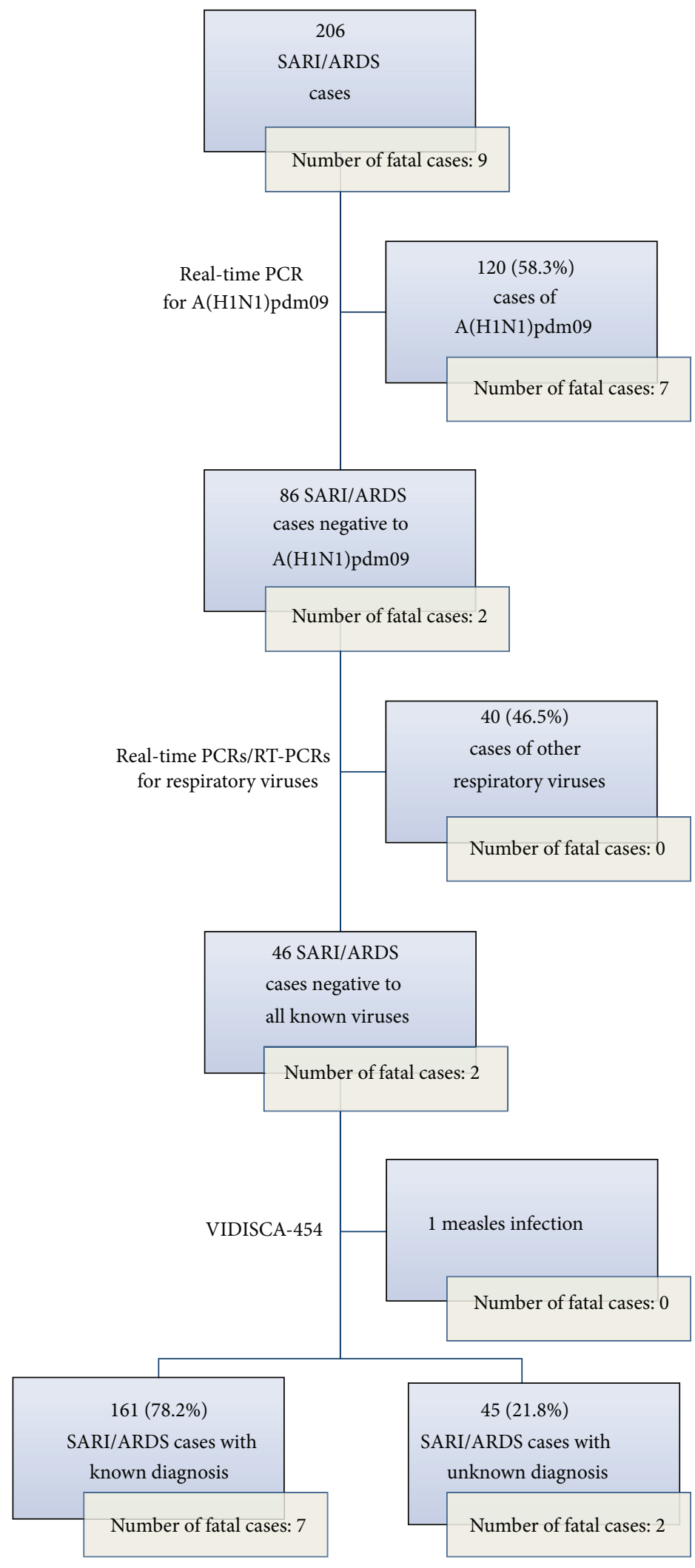

FIGURE 1: Study design and results overview. 
Forty-six (46/206: 22.3\%) SARI/ARDS cases (including two fatalities) resulted negative to all diagnostic assays $(58.2 \%$ males; $18.2 \%$ aged $\leq 5$ years, $45.4 \%$ aged $6-64$ years) and were further investigated by VIDISCA-454 [5, 11]. VIDISCA454 revealed no sequence reads that could belong to a novel virus or viral variant in any of the 46 specimens; however it enabled the identification of one case of undiagnosed measles, thus increasing the proportion of cases with a diagnosis to $78.2 \%(161 / 206)$. Hence, the overall proportion of cases with unknown diagnosis was $21.8 \%$ (45/206); most (34/45: $75.6 \%$ ) cases that could not be diagnosed were ARDS and two (2/45: $4.4 \%)$ were fatal. Figure 1 summarizes study results.

\section{Discussion}

During pandemic and postpandemic period, several pathogens cocirculated and were associated to severe respiratory infections; however, influenza A(H1N1)pdm09 virus had the greatest impact $(58.3 \%)$ in our SARI/ARDS series. More than half $(51.7 \%)$ of $\mathrm{A}(\mathrm{H} 1 \mathrm{~N} 1) \mathrm{pdm} 09$ infection resulted in ARDS. It is interesting to note that most $(67.5 \%)$ severe respiratory diseases due to $\mathrm{A}(\mathrm{H} 1 \mathrm{~N} 1) \mathrm{pdm} 09$ infection were identified among 6-64-year-old individuals. The A(H1N1)pdm09 case fatality rate in our ARDS series was $11.3 \%$ fatal cases in young adults, and $42.8 \%$ did not belong to any at-risk category $[3,4]$. This data is in agreement with other studies; Van Kerkhove et al. have reported a median age of 46 years among fatal laboratory-confirmed A(H1N1)pdm09 cases [12]. McCallum has described that during the 2009 pandemic only $1 \%$ of laboratory-confirmed cases and 13\% of laboratory-confirmed deaths were among persons 65 years of age or older [13]. The Global Pandemic Mortality (GLaMOR) project has evaluated that although the pandemic mortality estimate was similar in magnitude to that of seasonal influenza, a marked shift toward mortality among persons 65 years of age occurred, so that many more life-years were lost [14]. Such an age shift has been documented as well by several studies on A(H1N1)pdm09 mortality [15-17].

The proportion of SARI/ARDS cases associated with respiratory viruses other than $\mathrm{A}(\mathrm{H} 1 \mathrm{N1}) \mathrm{pdm} 09$ was significantly lower (19.4\% versus $58.3 \%, P$ value $<0.0000001)$. Severe respiratory diseases associated with respiratory viruses other than $\mathrm{A}(\mathrm{H} 1 \mathrm{~N} 1)$ pdm09 were detected more frequently among children $\leq 5$ years $(13.3 \%$ versus $30 \%, P$ value $=0.02)$. This piece of evidence is in accordance with the results of other studies reporting a notable burden of respiratory viruses in children under 5 years [18-20].

Studies published to date have suggested that influenza viruses and rhinoviruses are the leading causes of severe respiratory disease leading to hospitalization [21, 22], similarly to what was observed in our SARI/ARDS series, where $\mathrm{hRV} / \mathrm{hEV}$ were the most common identified viruses along with influenza viruses. Also influenza $A(\mathrm{H} 3 \mathrm{~N} 2)$ virus played a significant role in our SARI cases and caused ARDS in one patient with a weakened immune system due to HIV/AIDS. Overall, the proportion of SARI/ARDS correlated to an influenza A virus infection was $62.1 \%(128 / 206)$, thus emphasizing the central role of influenza A virus in severe respiratory infection $[23,24]$.

The use of molecular assay has notably contributed to identifying pathogens possibly involved in severe respiratory disease, thus allowing getting to a diagnosis of viral infection in nearly $80 \%$ of study SARI/ARDS cases. Other studies that have not used nucleic acid amplification assays have typically reported that $5-20 \%$ of cases of acute respiratory infection have a viral etiology [25]. In addition, it is noteworthy that VIDISCA-454 enabled the identification of one measles infection that escaped clinical diagnosis in one SARI case. Hence, measles infection should be considered in complicated pulmonary disease, as also suggested by others [26], since measles virus is not generally included in respiratory diagnostic panels.

\section{Conclusions}

During pandemic and postpandemic period, several pathogens cocirculated and were associated to severe respiratory infections, with influenza $\mathrm{A}(\mathrm{H} 1 \mathrm{~N} 1) \mathrm{pdm} 09$ virus having the greatest impact. Nearly $22 \%$ of SARI/ARDS cases did not obtain a definite diagnosis, and among these cases two were fatal. In clinical practice, great efforts should be devoted to improving diagnosis of severe respiratory infec-tions and to reducing such "diagnostic gap." The advantage from relying upon more accurate diagnosis could benefit the patient, in terms of receiving the more appropriate antiviral drugs, and could provide more detailed information on viruses circulating in the community, thus making public health authorities aware so as to adjust their policies accordingly.

VIDISCA-454 proved to be a sensitive and specific methodology that can be successfully applied to surveillance of viral respiratory infections that represent an ever-changing field due to the continuous emergence of new viruses (i.e., influenza $\mathrm{A}(\mathrm{H} 5 \mathrm{N1})$ and $\mathrm{A}(\mathrm{H} 7 \mathrm{~N} 9)$ viruses, MERS-CoV).

\section{Conflict of Interests}

The authors declare that there is no conflict of interests regarding the publication of this paper.

\section{Acknowledgments}

This study was funded by Grant no. 5988, 30/6/2011 from Direzione Generale Sanità, Regione Lombardia, and by the European Community's Seventh Framework Programme (FP7/2007-2013) under EMPERIE project, EC Grant agreement no. 223498 .

\section{References}

[1] P. Larussa, "Pandemic novel 2009 H1N1 influenza: what have we learned?" Seminars in Respiratory and Critical Care Medicine, vol. 32, no. 4, pp. 393-399, 2011.

[2] D. L. Swerdlow, L. Finelli, and C. B. Bridges, "2009 H1N1 influenza pandemic: field and epidemiologic investigations in the united states at the start of the first pandemic of the 21st 
century," Clinical Infectious Diseases, vol. 52, supplement 1, pp. S1-S3, 2011.

[3] European Center for Disease Control (ECDC), "Surveillance of severe disease due to influenza in Europe," http://www.ecdc .europa.eu/en/healthtopics/seasonal_influenza/Documents/ 1201_ECDC_concept_paper_Surveillance_of_severe_disease due_to_influenza_in_Europe.pdf.

[4] Italian Ministry of Health, "Surveillance of severe forms of influenza A(H1N1)pdm09 infection," http://www.trovanorme .salute.gov.it/normsan-pdf/0000/31217_1.pdf.

[5] M. de Vries, M. Deijs, M. Canuti et al., "A sensitive assay for virus discovery in respiratory clinical samples," PLoS ONE, vol. 6, no. 1, Article ID e16118, 2011.

[6] L. van der Hoek, K. Pyrc, M. F. Jebbink et al., "Identification of a new human coronavirus," Nature Medicine, vol. 10, no. 4, pp. 368-373, 2004.

[7] InfluNet, http://www.iss.it/iflu/.

[8] G. R. Bernard, A. Artigas, K. L. Brigham et al., "The AmericanEuropean Consensus Conference on ARDS: definitions, mechanisms, relevant outcomes, and clinical trial coordination," The American Journal of Respiratory and Critical Care Medicine I, vol. 149, no. 3, pp. 818-824, 1994.

[9] World Health Organization (WHO) Global Influenza Surveillance Network (GISN), "Manual for the laboratory diagnosis and virological surveillance of influenza," 2011, http:// whqlibdoc.who.int/publications/2011/9789241548090_eng.pdf.

[10] Center for Disease Control (CDC), "2009 protocol CDC protocol of real-time RT PCR for swine influenza A(H1N1)," 2009, http://www.who.int/csr/resources/publications/swineflu/ CDCrealtimeRTPCRprotocol_20090428.pdf.

[11] L. van der Hoek, M. de Vries, B. B. O. Munnink et al., "Performance of VIDISCA-454 in feces-suspensions and serum," Viruses, vol. 4, no. 8, pp. 1328-1334, 2012.

[12] M. D. van Kerkhove, K. A. H. Vandemaele, V. Shinde et al., "Risk factors for severe outcomes following 2009 influenza a (H1N1) infection: a global pooled analysis," PLoS Medicine, vol. 8, no. 7, Article ID e1001053, 2011.

[13] L. McCallum, "Epidemiological characteristics of the influenza A(H1N1)2009 pandemic in the Western Pacific Region," Western Pacific Surveillance and Response, vol. 1, pp. 5-11, 2010.

[14] L. Simonsen, P. Spreeuwenberg, R. Lustig, R. J. Taylor, D. M. Fleming, and M. Kroneman, "Global mortality estimates for the 2009 Influenza Pandemic from the GLaMOR project: a modeling study," PLoS Medicine, vol. 10, no. 11, Article ID e1001558, 2013.

[15] V. Charu, G. Chowell, L. S. P. Mejia et al., "Mortality burden of the A/H1N1 pandemic in Mexico: a comparison of deaths and years of life lost to seasonal influenza," Clinical Infectious Diseases, vol. 53, no. 10, pp. 985-993, 2011.

[16] M. Lemaitre, F. Carrat, G. Rey, M. Miller, L. Simonsen, and C. Viboud, "Mortality burden of the 2009 A/H1N1 influenza pandemic in France: comparison to seasonal influenza and the A/H3N2 pandemic," PLoS ONE, vol. 7, no. 9, Article ID e45051, 2012.

[17] D. J. Muscatello, M. A. Cretikos, and C. R. MacIntyre, "All-cause mortality during first wave of pandemic (H1N1) 2009, New South Wales, Australia, 2009," Emerging Infectious Diseases, vol. 16, no. 9, pp. 1396-1402, 2010.

[18] I. Rudan, O. 'Brien KL, H. Nair, L. Liu, E. Theodoratou, and S. Qazi, "Epidemiology and etiology of childhood pneumonia in
2010: estimates of incidence, severe morbidity, mortality, underlying risk factors and causative pathogens for 192 countries," Journal of Global Health, vol. 3, no. 1, Article ID 010401, 2013.

[19] C. L. F. Walker, I. Rudan, L. Liu et al., "Global burden of childhood pneumonia and diarrhoea," The Lancet, vol. 381, no. 9875, pp. 1405-1416, 2013.

[20] J. R. Verani, J. McCracken, W. Arvelo, A. Estevez, M. R. Lopez, and L. Reyes, "Surveillance for hospitalized acute respiratory infection in Guatemala," PLoS One, vol. 8, no. 12, Article ID e83600, 2013.

[21] L. C. Jennings, T. P. Anderson, K. A. Beynon et al., "Incidence and characteristics of viral community-acquired pneumonia in adults," Thorax, vol. 63, no. 1, pp. 42-48, 2008.

[22] M. A. Marcos, M. Camps, T. Pumarola et al., "The role of viruses in the aetiology of community-acquired pneumonia in adults," Antiviral Therapy, vol. 11, no. 3, pp. 351-359, 2006.

[23] T. Wiemken, P. Peyrani, K. Bryant et al., "Incidence of respiratory viruses in patients with community-acquired pneumonia admitted to the intensive care unit: results from the Severe Influenza Pneumonia Surveillance (SIPS) project," European Journal of Clinical Microbiology and Infectious Diseases, vol. 32, no. 5, pp. 705-710, 2013.

[24] S. H. Choi, S. B. Hong, G. B. Ko, Y. Lee, H. J. Park, and S. Y. Park, "Viral infection in patients with severe pneumonia requiring intensive care unit admission," The American Journal of Respiratory and Critical Care Medicine, vol. 186, pp. 325-332, 2012.

[25] T. M. File Jr., "Community-acquired pneumonia," The Lancet, vol. 362, no. 9400, pp. 1991-2001, 2003.

[26] A. H. L. Do, H. R. van Doorn, M. N. Nghiem et al., "Viral etiologies of acute respiratory infections among hospitalized vietnamese children in Ho Chi Minh City, 2004-2008," PLoS ONE, vol. 6, no. 3, Article ID e18176, 2011. 

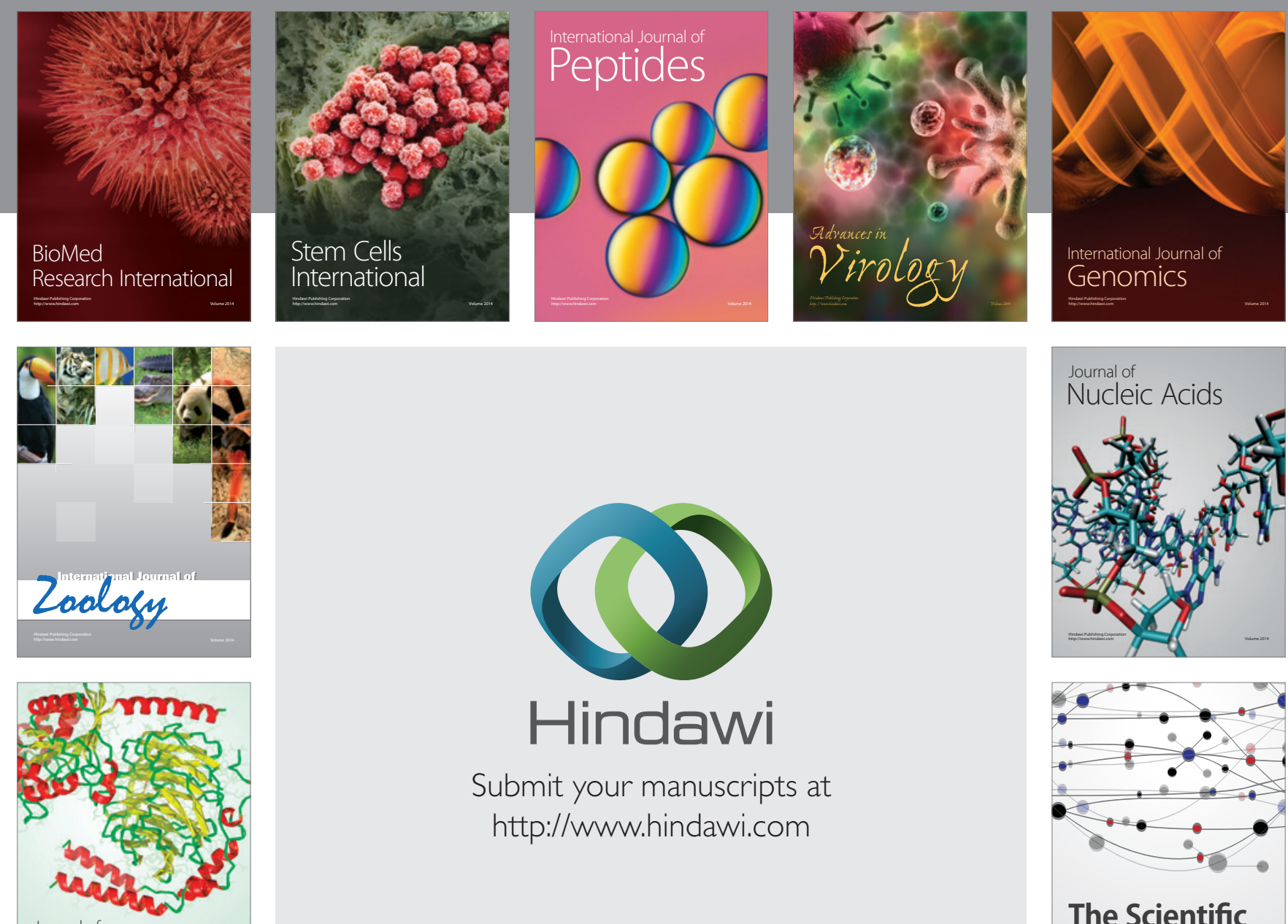

Submit your manuscripts at

http://www.hindawi.com

Journal of
Signal Transduction
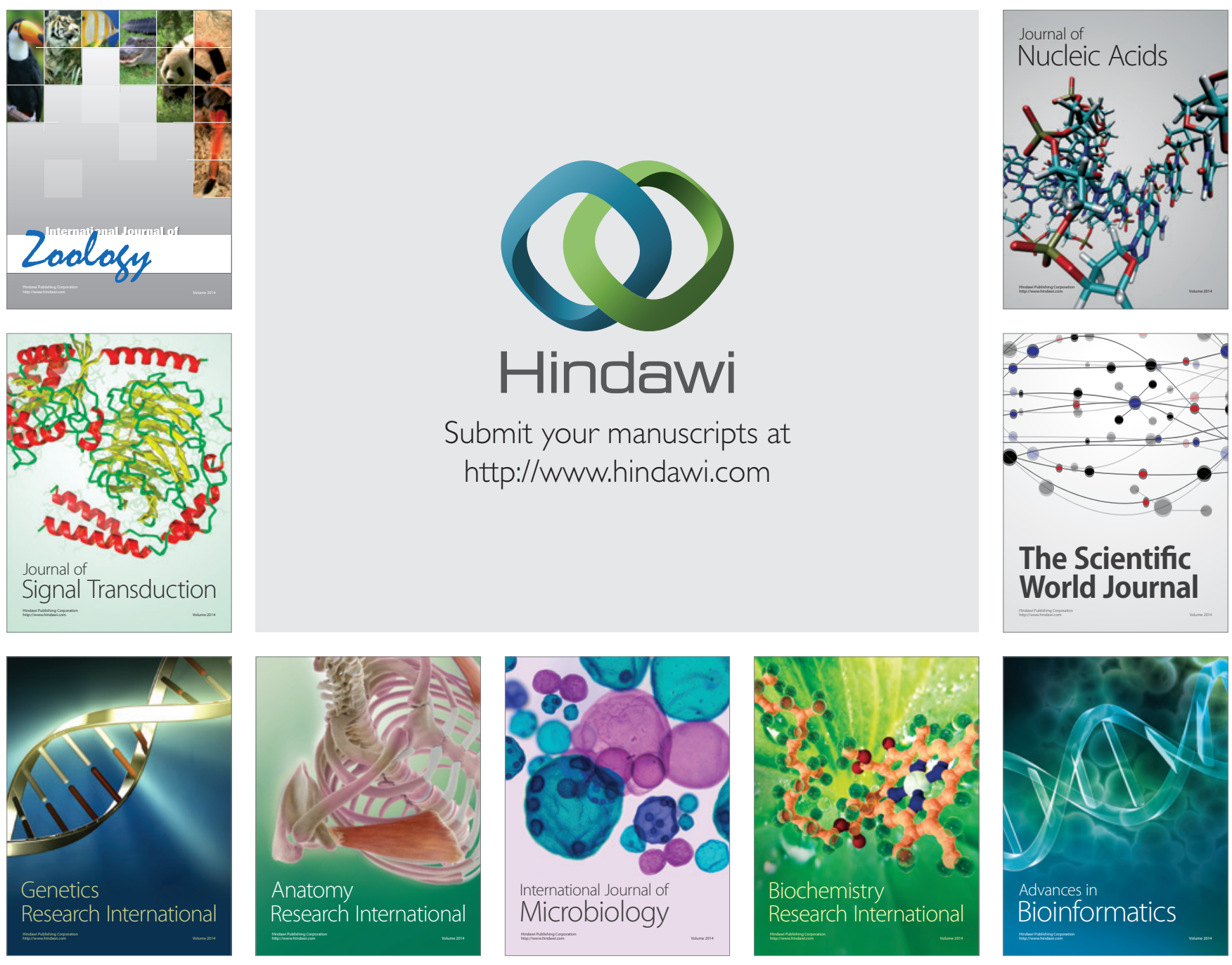

The Scientific World Journal
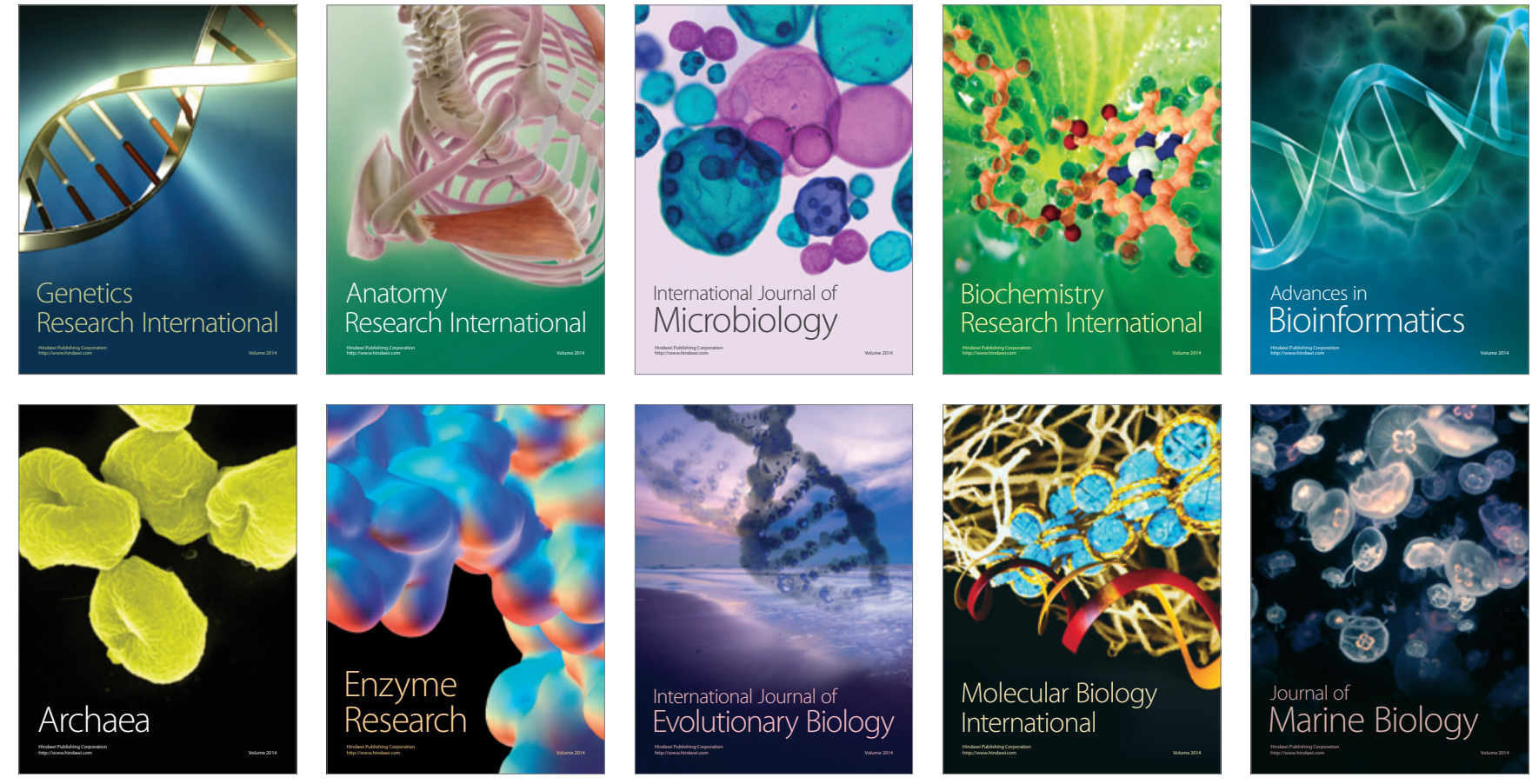